\title{
Unirationality of moduli spaces of special cubic fourfolds and K3 surfaces
}

\author{
Howard Nuer
}

\begin{abstract}
We provide explicit descriptions of the generic members of Hassett's divisors $\mathcal{C}_{d}$ in the moduli space $\mathcal{C}$ of smooth cubic fourfolds for relevant $18 \leqslant d \leqslant 38$ and for $d=44$. In doing so, we prove that $\mathcal{C}_{d}$ is unirational for these $d$. As a corollary, we prove that the moduli space $\mathcal{N}_{d}$ of polarized K3 surfaces of degree $d$ is unirational for $d=14,26,38$. The case $d=26$ is entirely new, while the other two cases have been previously proven by Mukai.
\end{abstract}

\section{Introduction}

In this paper, we systematically describe explicit surfaces that are contained in a generic special cubic fourfold of discriminant $d \leqslant 44$ with $d \neq 42$, recovering descriptions of the previously known cases for $d=12,14,20$. Recall that a smooth cubic fourfold $X \subset \mathbb{P}^{5}$ is the vanishing locus of a degree 3 homogeneous polynomial in 6 variables, and $X$ is called special if it contains the class of an algebraic surface $S$ not homologous to a complete intersection. Denoting the square of the hyperplane class by $h^{2}$, one defines the discriminant $d$ to be the discriminant of the saturated sublattice generated by $h^{2}$ and $S$. In [Has00], Hassett defined the sublocus of the moduli space $\mathcal{C}$ of smooth cubic fourfolds consisting of special cubic fourfolds of discriminant $d$, denoted $\mathcal{C}_{d}$. He proved that $\mathcal{C}_{d}$ is an irreducible divisor for any $d>6$ with $d \equiv 0,2(\bmod 6)$ and empty otherwise.

For the almost twenty years since Hassett's work, the only $\mathcal{C}_{d}$ whose generic member $X$ could be described explicitly were for $d=8,12,14$, and 20 . The surfaces $S$ in these cases were given by planes, cubic scrolls, quintic del Pezzos, and Veronese surfaces, respectively. For some of these choices of $d$, the generic $X \in \mathcal{C}_{d}$ admits an alternative description. For example, the generic $X \in \mathcal{C}_{8}$ can be described as containing an octic K3 surface, and the generic $X \in \mathcal{C}_{14}$ contains a quartic scroll. It is nevertheless notable that for each $d$ above, the surface $S$ can be taken to be a smooth rational surface. By using the deformation theory of Hilbert schemes of flags and appropriately chosen linear systems on rational surfaces, we show that this trend continues for all relevant $d \leqslant 38$. For $d=44$, however, we obtain a different kind of description.

Theorem 1.1. The generic element of $\mathcal{C}_{d}$ for $12 \leqslant d \leqslant 38$ contains a smooth rational surface obtained as the blow-up of $\mathbb{P}^{2}$ at $p$ generic points and embedded into $\mathbb{P}^{5}$ via the very ample linear

Received 13 August 2015, accepted in final form 23 July 2016.

2010 Mathematics Subject Classification 14C05 (primary); 14D07, 14D20, 14J26, 14J28, $14 \mathrm{E} 08$ (secondary).

Keywords: Hodge theory, cubic fourfolds, Enriques surfaces, Coble surfaces.

This journal is (C) Foundation Compositio Mathematica 2017. This article is distributed with Open Access under the terms of the Creative Commons Attribution Non-Commercial License, which permits non-commercial reuse, distribution, and reproduction in any medium, provided that the original work is properly cited. For commercial re-use, please contact the Foundation Compositio Mathematica.

The author was partially supported by NSF grant DMS 1201466. 


\section{H. NUER}

system $|H|=\left|a L-\left(E_{1}+\cdots+E_{i}\right)-2\left(E_{i+1}+\cdots+E_{i+j}\right)-3\left(E_{i+j+1}+\cdots+E_{p}\right)\right|$, where $H$ is given by Table 1. Moreover, the generic $X \in \mathcal{C}_{44}$ contains a Fano embedded Enriques surface.

The problem of concretely describing the generic $X \in \mathcal{C}_{d}$ is interesting in its own right, and it is only natural to attempt to describe the cubics in $\mathcal{C}_{d}$ in terms of their geometry. But the problem is also intimately related to the geometry of the $\mathcal{C}_{d}$ themselves. Indeed, $\mathcal{C}_{d}$ is known to be unirational for $d=8,12,14,20$, for example, precisely because the cubics they parametrize contain the specific surfaces mentioned above. The method we use to prove Theorem 1.1 above gives unirationality as a consequence.

Theorem 1.2. For $12 \leqslant d \leqslant 44$ with $d \neq 42$, the moduli space $\mathcal{C}_{d}$ is unirational.

Unirationality of a moduli space is a very useful property. It indicates that the generic element can be written down explicitly in free coordinates.

One expects that $\mathcal{C}_{d}$ ceases to be unirational as $d$ grows, and it is natural to ask what is the smallest $d$ such that $\mathcal{C}_{d}$ is not unirational, and at the other extreme, one can ask if there is a minimal $d$ after which $\mathcal{C}_{d}$ is of general type. Questions of this nature have been previously investigated by Gritsenko, Hulek, and Sankaran in the cases of polarized K3 surfaces and certain families of holomorphic symplectic manifolds (see [GHS13] for a good account). They prove, for example, that the moduli space $\mathcal{N}_{2 d}$ of polarized K3 surfaces of degree $2 d$ has non-negative Kodaira dimension for $d \geqslant 40$ with $d \neq 41,44,45,47$ and is of general type for $d>61$, as well as for $d=46,50,52,54,57,58,60$. By using a surprising and beautiful connection between the period domains of cubic fourfolds and polarized K3 surfaces [Has00], one can translate this result about $\mathcal{N}_{d}$ to get the following.

Proposition 1.3. Let $d>80$ with $d \equiv 2(\bmod 6)$ and $4 \nmid d$ be such that for any odd prime $p$, we have that $p \mid d$ implies $p \equiv 1(\bmod 3)$. Then the Kodaira dimension of $\mathcal{C}_{d}$ is non-negative. If moreover $d>122$, then $\mathcal{C}_{d}$ is of general type.

Proposition 1.3 thus provides an infinite number of large $d$ such that $\mathcal{C}_{d}$ is of general type, and one expects that the gaps can be filled in using automorphic form techniques as in the K3 case. This has recently been shown to be the case by Várilly-Alvarado and Tanimoto [TV15]. ${ }^{1}$ The results of [TV15] bound from above the minimum discriminant required for $\mathcal{C}_{d}$ to have non-negative Kodaira dimension, and Theorem 1.2 gives 44 as a lower bound.

Through the isomorphism of period domains associated with $\mathcal{N}_{d}$ and $\mathcal{C}_{d}$ for special values of $d$ [Has00], Theorem 1.2 implies the following result.

Theorem 1.4. The moduli space $\mathcal{N}_{d}$ parametrizing polarized K3 surfaces of degree $d$ is unirational for $d=14,26,38$.

The cases $d=14,38$ were already known due to the work of Mukai. He obtains his results by presenting the generic $T \in \mathcal{N}_{d}$ as a complete intersection in a certain homogeneous space. The case $d=26$ is entirely new as far as I know and fills in a long-standing gap in known unirationality results $\left(\mathcal{N}_{d}\right.$ was already known to be unirational for $2 \leqslant d \leqslant 24$ and $d=30,32,34,38$; for $d=26$, however, $\mathcal{N}_{26}$ was shown to have negative Kodaira dimension by Peterson [Pet15]). While this result proves the unirationality of $\mathcal{N}_{26}$, it does not provide a geometric construction of the generic K3 surface it parametrizes. Such a construction remains an interesting open problem.

\footnotetext{
${ }^{1}$ In particular, they have shown that $\mathcal{C}_{6 n+2}$ is of general type for $n>18$ and $n \neq 20,21,25$ and has non-negative Kodaira dimension for $n>13$ and $n \neq 15$. Moreover, $\mathcal{C}_{6 n}$ is of general type for $n>18$ and $n \neq 20,22,23,25,30,32$, and it has non-negative Kodaira dimension for $n>16$ and $n \neq 18,20,22,30$.
} 


\section{UNIRATIONALITY OF MODULI SPACES}

\section{Review: cubic fourfolds and the divisors $\mathcal{C}_{d} \subset \mathcal{C}$}

Let $X \subset \mathbb{P}^{5}$ be a smooth cubic fourfold, and define $A(X):=H^{2,2}(X) \cap H^{4}(X, \mathbb{Z})$. In $[\operatorname{Has} 00]$, Hassett studied special cubic fourfolds, where a cubic fourfold $X$ is special if $\operatorname{rk} A(X) \geqslant 2$. Inside the coarse moduli space $\mathcal{C}$ of smooth cubic fourfolds, which is defined as the GIT quotient $\mathcal{C}:=\mathbb{P} H^{0}\left(\mathbb{P}^{5}, \mathcal{O}(3)\right)_{\text {smooth }} / / \mathrm{PGL}_{6}$, a very general cubic fourfold $X$ satisfies $\mathrm{rk} A(X)=1$. Define the rank 2 lattice $K_{d}$ to be



and let $\mathcal{C}_{d}$ be the locus of $X \in \mathcal{C}$ for which $K_{d}$ is a saturated sublattice of $A(X)$ containing $h^{2}$. Then the main theorem we need is the following.

Theorem 2.1 ([Has00, Theorem 1.0.1]). If a cubic fourfold $X$ is special, then $[X] \in \mathcal{C}_{d}$ for some $d$. Moreover, $\mathcal{C}_{d} \neq \varnothing$ if and only if $d>6$ is an integer with $d \equiv 0,2(\bmod 6)$, in which case $\mathcal{C}_{d}$ is an irreducible algebraic divisor of $\mathcal{C}$.

\section{Explicit descriptions of the divisors $\mathcal{C}_{d}$ for $d \leqslant 38$ and $d=44$}

Hassett provided an explicit description of the generic members of the divisors $\mathcal{C}_{d}$ for $d \leqslant 20$ with $d \neq 18$. In this section, we find explicit descriptions of the generic members of the divisors $\mathcal{C}_{d}$ for $18 \leqslant d \leqslant 38$ and $d=44$. In fact, our methods recover all of the previously known results. Our main tools are the deformation theory of Hilbert schemes of flags, semicontinuity arguments, and explicit Macaulay2 calculations. ${ }^{2}$

Consider the Flag Hilbert scheme

$$
\mathbf{F H}=\{(S, X) \mid S \subset X\} \subset \operatorname{Hilb}_{\mathbb{P}^{5}}^{\chi\left(\mathcal{O}_{S}(n)\right)} \times V,
$$

where $S$ a smooth surface and $V \subset \mathbb{P}^{55}$ parametrizes smooth cubic fourfolds, and its two projections

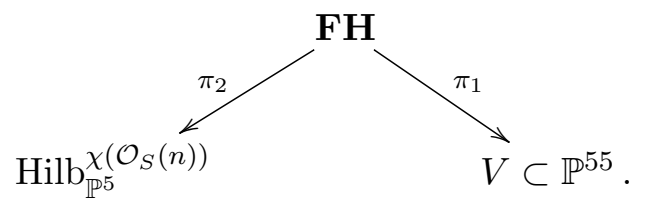

The first projection $\pi_{1}: \mathbf{F H} \rightarrow \operatorname{Hilb}_{\mathbb{P}^{5}}^{\chi\left(\mathcal{O}_{S}(n)\right)}$ sends a flag $(S \subset X)$ to the corresponding point $[S]$ in the Hilbert scheme $\operatorname{Hilb}_{\mathbb{P}^{5}}^{\chi\left(\mathcal{O}_{S}(n)\right)}$. The fiber of this morphism above $[S]$ is $\mathbb{P}\left(H^{0}\left(\mathcal{I}_{S / \mathbb{P}^{5}}(3)\right)\right)$. By the semicontinuity theorem, $h^{0}\left(\mathcal{I}_{S / \mathbb{P}^{5}}(3)\right)$ achieves its minimum value on an open subset

${ }^{2}$ Scripts for duplicating the computer calculations performed in this paper are available at the author's website http://www. math.rutgers. edu/ hjn11/publications/index.html. 


\section{H. NUER}

$W \subset \operatorname{Hilb}_{\mathbb{P}^{5}}^{\chi\left(\mathcal{O}_{S}(n)\right)}$. If we assume that $\operatorname{Hilb}_{\mathbb{P}^{5}}^{\chi\left(\mathcal{O}_{S}(n)\right)}$ is smooth at $[S] \in W$, so that

$$
\operatorname{dim}_{[S]} \operatorname{Hilb}_{\mathbb{P}^{5}}^{\chi\left(\mathcal{O}_{S}(n)\right)}=\operatorname{dim} T_{[S]} \operatorname{Hilb}_{\mathbb{P}^{5}}^{\chi\left(\mathcal{O}_{S}(n)\right)}=h^{0}\left(S, N_{S / \mathbb{P}^{5}}\right)
$$

by standard deformation theory, the open subset $\pi_{1}^{-1}(W) \subset \mathbf{F H}$ is a projective bundle over $W$, with fibers isomorphic to $\mathbb{P}^{h^{0}\left(\mathcal{I}_{S / \mathbb{P}^{5}}(3)\right)-1}$, and is smooth around the fiber $\pi_{1}^{-1}([S])$. Thus for any cubic fourfold $X \supset S$, we have by (3.1) that

$$
\operatorname{dim}_{(S \subset X)} \mathbf{F H}=h^{0}\left(S, N_{S / \mathbb{P}^{5}}\right)+h^{0}\left(\mathcal{I}_{S / \mathbb{P}^{5}}(3)\right)-1 .
$$

Now, consider the second projection $\pi_{2}: \mathbf{F H} \rightarrow V$. As those cubic fourfolds $X$ lying in the image of $\pi_{2}$ are special, the image of $\pi_{2}$ must be contained in the union of the preimages of the $\mathcal{C}_{d}$ under the quotient map $V \rightarrow V / / \mathrm{PGL}_{6}=\mathcal{C}$ and thus has dimension at most $54=$ $\operatorname{dim} \mathcal{C}_{d}+\operatorname{dim} \mathrm{PGL}_{6}$. It follows from the theorem on the dimension of fibers that the fibers of $\left.\pi_{2}\right|_{\pi_{1}^{-1}(W)}$ must satisfy

$$
\operatorname{dim} \pi_{2}^{-1}(X) \geqslant \operatorname{dim} \mathbf{F H}-\operatorname{dim} \pi_{2}(\mathbf{F H}) \geqslant \operatorname{dim} \mathbf{F H}-54=h^{0}\left(S, N_{S / \mathbb{P}^{5}}\right)+h^{0}\left(\mathcal{I}_{S / \mathbb{P}^{5}}(3)\right)-55,
$$

where the last equality follows from (3.2). By this inequality, we get

$$
h^{0}\left(S, N_{S / X}\right) \geqslant \operatorname{dim} \pi_{2}^{-1}(X) \geqslant h^{0}\left(S, N_{S / \mathbb{P}^{5}}\right)+h^{0}\left(\mathcal{I}_{S / \mathbb{P}^{5}}(3)\right)-55
$$

because $\pi_{2}^{-1}(X)=\operatorname{Hilb}_{X}^{\chi\left(\mathcal{O}_{S}(n)\right)}$, which has tangent space $H^{0}\left(S, N_{S / X}\right)$.

Thus, if we can exhibit a specific flag $(S \subset X)$ such that $h^{0}\left(\mathcal{I}_{S / \mathbb{P}^{5}}(3)\right)$ is minimal, Hilb $\left.\mathbb{P}^{5} \mathcal{O}_{S}(n)\right)$ is smooth at $[S]$, and $h^{0}\left(S, N_{S / X}\right)=h^{0}\left(S, N_{S / \mathbb{P}^{5}}\right)+h^{0}\left(\mathcal{I}_{S / \mathbb{P}^{5}}(3)\right)-55$, then we must have equality everywhere in (3.3) and (3.2). For the irreducible component $Z \subset W$ containing [S], it follows that the closure $\overline{\pi_{2}\left(\pi_{1}^{-1}(Z)\right)}$ is an irreducible divisor. By computing the self-intersection of $S \subset X$ using the formula from [Has00, Section 4.1],

$$
S^{2}=c_{2}\left(N_{S / X}\right)=6 H^{2}+3 H \cdot K_{S}+K_{S}^{2}-\chi_{S}
$$

in each of our cases, we can show that $\overline{\pi_{2}\left(\pi_{1}^{-1}(Z)\right)}=\mathcal{C}_{d}$ for $d=3 S^{2}-\left(H^{2}\right)^{2}$. Note that here $H=c_{1}\left(\mathcal{O}_{S}(1)\right)$.

We use Macaulay2 to exhibit such flags for each $18 \leqslant d \leqslant 38$ and $d=44$. The case $d=44$ is exceptional, and we deal with it separately. First, let $S$ be the blow-up of $\mathbb{P}^{2}$ at $p$ points in general position, and denote by $E_{m}$ for $m=1, \ldots, p$ the exceptional divisors. Then $K_{S}^{2}=9-p$ and $\chi(S)=$ $3+p$. Consider divisors of the form $H=a L-\left(E_{1}+\cdots+E_{i}\right)-2\left(E_{i+1}+\cdots+E_{i+j}\right)-3\left(E_{i+j+1}+\right.$ $\left.\cdots+E_{p}\right)$ with $\operatorname{deg} S=H^{2}$, where $L$ is the pull-back of the hyperplane class on $\mathbb{P}^{2}$. For each $12 \leqslant d \leqslant 38$ such that $\mathcal{C}_{d} \neq \varnothing$, we choose the polarization $H$ according to Table 1 . For each $d$, we present the very ample divisor with lowest value of $a$ on a given surface $S$ (that is, for a fixed $p$ ). ${ }^{3}$

By choosing $p$ random points in $\mathbb{P}^{2}\left(\mathbb{F}_{q}\right)$ for a large prime $q$, one can verify in Macaulay2 that the linear system $|H|=\left|a L-\left(E_{1}+\cdots+E_{i}\right)-2\left(E_{i+1}+\cdots+E_{i+j}\right)-3\left(E_{i+j+1}+\cdots+E_{p}\right)\right|$ embeds the blow-up $S$ as a linearly normal smooth surface in $\mathbb{P}^{5}$ of degree $H^{2}$ and thus is very ample. It is easy to see that the blow-up $S$ satisfies $h^{1}\left(N_{S / \mathbb{P}^{5}}\right)=0$, so the Hilbert scheme $\operatorname{Hilb}_{\mathbb{P}^{5}}^{\chi\left(\mathcal{O}_{S}(n)\right)}$ is smooth at $[S]$, of dimension $h^{0}\left(N_{S / \mathbb{P}^{5}}\right)=2 p+27$. Furthermore, we find that for a random choice of these $p$ points and for a random choice of cubic fourfold $X \in \mathbb{P} H^{0}\left(\mathcal{I}_{S / \mathbb{P}^{5}}(3)\right)$, the dimensions $h^{0}\left(N_{S / X}\right)$ and $h^{0}\left(\mathcal{I}_{S / \mathbb{P}^{5}}(3)\right)$ achieve the values reported in Table 1.

\footnotetext{
${ }^{3}$ For some choices of $d$ and $p$, we found multiple very ample polarizations giving the same numerical and cohomological invariants. Upon inspection, these were usually found to be equivalent by thinking of $S$ as a blow-up of $\mathbb{P}^{2}$ at $p$ points in a different way.
} 


\section{UNIRATIONALITY OF MODULI SPACES}

From the long exact sequence on cohomology associated with the short exact sequence

$$
0 \rightarrow \mathcal{I}_{S / \mathbb{P}^{5}}(3) \rightarrow \mathcal{O}_{\mathbb{P}^{5}}(3) \rightarrow \mathcal{O}_{S}(3) \rightarrow 0
$$

and a Riemann-Roch calculation, these values of $h^{0}\left(\mathcal{I}_{S / \mathbb{P}^{5}}(3)\right)$ can be seen to be the smallest possible. Furthermore, in each case we see that $h^{0}\left(S, N_{S / X}\right)=h^{0}\left(S, N_{S / \mathbb{P}^{5}}\right)+h^{0}\left(\mathcal{I}_{S / \mathbb{P}^{5}}(3)\right)-55$, as required. By the openness of smoothness and very ampleness, it follows that all of the above holds true for a generic choice of $p$ points in $\mathbb{P}^{2}$ over $\mathbb{C}$ and a generic choice of cubic in $H^{0}\left(\mathcal{I}_{S / \mathbb{P}^{5}}(3)\right)$. For more on this style of proof in the case of moduli of curves, see [Sch13].

We have thus proven the following theorem.

THEOREM 3.1. The generic element of $\mathcal{C}_{d}$ for $12 \leqslant d \leqslant 38$ contains a smooth rational surface obtained as the blow-up of $\mathbb{P}^{2}$ at $p$ generic points and embedded into $\mathbb{P}^{5}$ via the very ample linear system $|H|=\left|a L-\left(E_{1}+\cdots+E_{i}\right)-2\left(E_{i+1}+\cdots+E_{i+j}\right)-3\left(E_{i+j+1}+\cdots+E_{p}\right)\right|$, where $H$ is given by Table 1.

Now, let us treat the case $d=44$. We consider Fano models of Enriques surfaces. These are given by very ample polarizations $\Delta$ with $\Delta^{2}=10$ and $\Delta \cdot F \geqslant 3$ for every effective $F$ with $F^{2}=0$. Every Enriques surface $S$ admits such a polarization, and these polarizations embed $S$ into $\mathbb{P}^{5}$ as a surface of degree 10 whose homogeneous ideal is generated by ten cubics. It is well known that the Hilbert scheme $\mathrm{Hilb}_{\mathbb{P}^{5}}^{5 n^{2}+1}$ contains a smooth open subset of dimension 45 parametrizing Fano models of Enriques surfaces [CD85]. It follows that we get a smooth open subset of $\mathbf{F H}$ of dimension 54. By again taking a random Fano Enriques $S$ and cubic fourfold $X$ containing it, one finds that $h^{0}\left(N_{S / X}\right)=0$. This proves the following.

Theorem 3.2. The generic element of $\mathcal{C}_{44}$ contains a Fano model of an Enriques surface.

The cases $(d, p)=(30,10)$ and $(d, p)=(38,10)$ are intimately related to each other and to the case $d=44$ and have their origin in some very classical algebraic geometry. If we allow the K3 cover of an Enriques surface to develop an ordinary double point (ODP) fixed by the involution, then the resulting quotient surface has a quartic singularity whose resolution is an irreducible smooth rational curve $D_{0}$ with self-intersection -4 . The resulting surface $S$, known as a Coble surface, can be realized as the blow-up of $\mathbb{P}^{2}$ at the ten nodes of a rational planar sextic, the proper transform of which is $D_{0} \in\left|-2 K_{S}\right|$. These nodal curves, called Coble curves, were studied extensively at the beginning of the twentieth century by Coble. Analogous to the theory of Fano polarizations for Enriques surfaces, one can define Fano polarizations for Coble surfaces, and the divisor $H=10 L-3\left(E_{1}+\cdots+E_{10}\right)$ is the standard one. The linear system $|H|$ maps the Coble surface to $\mathbb{P}^{5}$ as a degree 10 surface while contracting $D_{0}$. Deforming the ten points into general position ensures that $\left|-m K_{S}\right|=\varnothing$ for any $m \geqslant 1$, so that $|H|$ contracts no curves and thus is ample. We proved in Theorem 3.1 that $|H|$ is in fact very ample, and by considering the cubics containing these "generalized" Coble surfaces, we obtained the first description of $\mathcal{C}_{38}$ above. The cubic fourfolds which contain the image of the genuine Coble surfaces form an irreducible divisor contained in the boundary of $\mathcal{C}_{38}$, as they generically admit a single ODP (and thus are rational). The adjoint Fano embedding, $\left|H+K_{S}\right|=\left|7 L-2\left(E_{1}+\cdots+E_{10}\right)\right|$, gives the above description of $\mathcal{C}_{30}$. The locus of cubic fourfolds containing the genuine Coble surfaces is again a divisor in this case, but this divisor is no longer only contained in the boundary and in fact parametrizes smooth cubic fourfolds. Viewed together as subvarieties of $\mathbb{P}^{5}$, these three cases form part of a larger story involving the irreducible components of the same Hilbert scheme, which we hope to come back to elsewhere. 
TABLE 1. Smooth rational surfaces

\begin{tabular}{|c|c|c|c|c|c|c|c|}
\hline$d$ & $p$ & $H$ & $H^{2}$ & $H \cdot K_{S}$ & $h^{0}\left(\mathcal{I}_{S / \mathbb{P}^{5}}(3)\right)$ & $h^{0}\left(N_{S / \mathbb{P}^{5}}\right)$ & $h^{0}\left(N_{S / X}\right)$ \\
\hline 12 & 7 & $4 L-\left(E_{1}+\cdots+E_{6}\right)-3 E_{7}$ & 6 & -4 & 22 & 41 & 8 \\
\hline 12 & 13 & $5 L-\left(E_{1}+\cdots+E_{12}\right)-2 E_{13}$ & 9 & -1 & 13 & 53 & 11 \\
\hline 12 & 16 & $7 L-\left(E_{1}+\cdots+E_{9}\right)-2\left(E_{10}+\cdots+E_{16}\right)$ & 12 & 2 & 4 & 59 & 8 \\
\hline 14 & 4 & $3 L-E_{1}-E_{2}-E_{3}-E_{4}$ & 5 & -5 & 25 & 35 & 5 \\
\hline 14 & 9 & $4 L-E_{1}-\cdots-E_{9}$ & 7 & -3 & 19 & 45 & 9 \\
\hline 14 & 11 & $5 L-\left(E_{1}+\cdots+E_{9}\right)-2\left(E_{10}+E_{11}\right)$ & 8 & -2 & 16 & 49 & 10 \\
\hline 14 & 14 & $6 L-\left(E_{1}+\cdots+E_{10}\right)-2\left(E_{11}+\cdots+E_{14}\right)$ & 10 & 0 & 10 & 55 & 10 \\
\hline 14 & 15 & $7 L-\left(E_{1}+\cdots+E_{9}\right)-2\left(E_{10}+\cdots+E_{14}\right)-3 E_{15}$ & 11 & 1 & 7 & 57 & 9 \\
\hline 14 & 16 & $8 L-\left(E_{1}+\cdots+E_{6}\right)-2\left(E_{7}+\cdots+E_{15}\right)-3 E_{16}$ & 13 & 3 & 1 & 59 & 5 \\
\hline 18 & 12 & $6 L-\left(E_{1}+\cdots+E_{7}\right)-2\left(E_{8}+\cdots+E_{12}\right)$ & 9 & -1 & 13 & 51 & 9 \\
\hline 18 & 15 & $8 L-\left(E_{1}+\cdots+E_{6}\right)-2\left(E_{7}+\cdots+E_{13}\right)-3\left(E_{14}+E_{15}\right)$ & 12 & 2 & 4 & 57 & 6 \\
\hline 20 & 0 & $2 L$ & 4 & -6 & 28 & 27 & 0 \\
\hline 20 & 10 & $6 L-\left(E_{1}+\cdots+E_{4}\right)-2\left(E_{5}+\cdots+E_{10}\right)$ & 8 & -2 & 16 & 47 & 8 \\
\hline 20 & 13 & $7 L-\left(E_{1}+\cdots+E_{6}\right)-2\left(E_{7}+\cdots+E_{12}\right)-3 E_{13}$ & 10 & 0 & 10 & 53 & 8 \\
\hline 20 & 14 & $7 L-\left(E_{1}+\cdots+E_{6}\right)-2\left(E_{7}+\cdots+E_{14}\right)$ & 11 & 1 & 7 & 55 & 7 \\
\hline 20 & 15 & $8 L-\left(E_{1}+E_{2}+E_{3}\right)-2\left(E_{4}+\cdots+E_{15}\right)$ & 13 & 3 & 1 & 57 & 3 \\
\hline 24 & 11 & $7 L-\left(E_{1}+\cdots+E_{3}\right)-2\left(E_{4}+\cdots+E_{10}\right)-3 E_{11}$ & 9 & -1 & 13 & 49 & 7 \\
\hline 24 & 14 & $8 L-\left(E_{1}+E_{2}+E_{3}\right)-2\left(E_{4}+\cdots+E_{13}\right)-3 E_{14}$ & 12 & 2 & 4 & 55 & 4 \\
\hline 26 & 12 & $7 L-\left(E_{1}+E_{2}+E_{3}\right)-2\left(E_{4}+\cdots+E_{12}\right)$ & 10 & 0 & 10 & 51 & 6 \\
\hline 26 & 13 & $8 L-\left(E_{1}+E_{2}+E_{3}\right)-2\left(E_{4}+\cdots+E_{11}\right)-3\left(E_{12}+E_{13}\right)$ & 11 & 1 & 7 & 53 & 5 \\
\hline 30 & 10 & $7 L-2\left(E_{1}+\cdots+E_{10}\right)$ & 9 & -1 & 13 & 47 & 5 \\
\hline 32 & 11 & $9 L-E_{1}-2\left(E_{2}+\cdots+E_{5}\right)-3\left(E_{6}+\cdots+E_{11}\right)$ & 10 & 0 & 10 & 49 & 4 \\
\hline 36 & 12 & $10 L-2\left(E_{1}+\cdots+E_{4}\right)-3\left(E_{5}+\cdots+E_{12}\right)$ & 12 & 2 & 4 & 51 & 0 \\
\hline 38 & 10 & $10 L-3\left(E_{1}+\cdots+E_{10}\right)$ & 10 & 0 & 10 & 47 & 2 \\
\hline 38 & 11 & $10 L-2\left(E_{1}+E_{2}\right)-3\left(E_{3}+\cdots+E_{11}\right)$ & 11 & 1 & 7 & 49 & 1 \\
\hline
\end{tabular}




\section{UNIRATIONALITY OF MODULI SPACES}

\section{Unirationality of some $\mathcal{C}_{d}$}

From the proof of Theorem 3.1 above, we see that for $12 \leqslant d \leqslant 38$, there is an open subset $U_{d} \subset\left(\mathbb{P}^{2}\right)^{p}$ parametrizing generic $p$-tuples of distinct points giving the cohomological invariants in Table 1. Moreover, there is a vector bundle $V_{d} \rightarrow U_{d}$ whose fiber over $\left(x_{1}, \ldots, x_{p}\right)$ is the vector space $H^{0}\left(\mathcal{I}_{S / \mathbb{P}^{5}}(3)\right)$, where $S$ is the blow-up of $\mathbb{P}^{2}$ at the $p$ points $x_{1}, \ldots, x_{p}$ and the embedding into $\mathbb{P}^{5}$ is given by $\left|a L-\left(E_{1}+\cdots+E_{i}\right)-2\left(E_{i+1}+\cdots+E_{i+j}\right)-3\left(E_{i+j+1}+\cdots+E_{p}\right)\right|$. Finally, Theorem 3.1 shows that the natural morphism $\mathbb{P}\left(V_{d}\right) \rightarrow \mathcal{C}_{d}$ is dominant. From the rationality of $\mathbb{P}\left(V_{d}\right)$, we get the following result.

Corollary 4.1. For $12 \leqslant d \leqslant 38$, the moduli space $\mathcal{C}_{d}$ is unirational.

Similarly, for $d=44$, we found a vector bundle $V_{44}$ over the moduli space of Fano polarized Enriques surfaces with fibers $H^{0}\left(\mathcal{I}_{S / \mathbb{P}^{5}}(3)\right)$ such that the natural moduli morphism $\mathbb{P}\left(V_{44}\right) \rightarrow \mathcal{C}_{44}$ is generically finite and dominant. Verra proved in [Ver84] that the moduli space of Fano polarized Enriques surfaces is unirational. From the generic finiteness and dominance of the morphism $\mathbb{P}\left(V_{44}\right) \rightarrow \mathcal{C}_{44}$, the next statement follows as in [Uen75, Theorem 6.10].

Corollary 4.2. The moduli space $\mathcal{C}_{44}$ is unirational.

\section{Unirationality of $\mathcal{N}_{d}$ for $d=14,26,38$}

In addition to describing the $\mathcal{C}_{d}$ and providing conditions for their non-emptiness, Hassett determined in [Has00] for which $d$ there exists a degree $d$ polarized K3 surface $(T, f)$ such that the primitive cohomology $H^{2}(T, \mathbb{C})^{0}:=(f)^{\perp} \subset H^{2}(T, \mathbb{C})$ is equivalent to the non-special cohomology $W_{X, K_{d}}:=K_{d}^{\perp}$, where $X \in \mathcal{C}_{d}$ and $K_{d}$ is the corresponding sublattice of discriminant $d$. In this case, we say that $X$ has an associated $\mathrm{K} 3$ surface, namely $(T, f)$. Hassett proved the following important result.

Theorem 5.1 ([Has00, Theorem 5.2.1]). A cubic fourfold $X \in \mathcal{C}_{d}$ has an associated K3 surface if and only if $4 \nmid d$ and $9 \nmid d$, and any odd prime $p$ such that $p \mid d$ satisfies $p \equiv 0,1(\bmod 3)$.

In fact, Hassett proved a bit more. For those $d$ as in Theorem 5.1, Hassett proved that the moduli spaces $\mathcal{C}_{d}$ and $\mathcal{N}_{d}$ are related. In particular, he showed that there is a rational map $\mathcal{N}_{d} \rightarrow \mathcal{C}_{d}$ which birational if $d \equiv 2(\bmod 6)$ and a double cover if $d \equiv 0(\bmod 6)$ [Has00, Section 5.3]. For $d=14,26,38$, every $X \in \mathcal{C}_{d}$ has an associated K3 surface, and the corresponding moduli spaces are birational. Using Corollary 4.1, we prove the unirationality of the corresponding moduli spaces of K3 surfaces.

TheOREM 5.2. For $d=14,26,38$, the moduli space of polarized K3 surfaces of degree $d$ is unirational.

\section{Final comments and open questions}

\subsection{What makes special cubic fourfolds special?}

It is worth noting that Voisin has shown that for a special cubic fourfold $X$, the lattice $A(X)$ can be generated either by smooth surfaces [Voi14, proof of Theorem 5.6] or by possibly singular rational surfaces [Voi07], and many have wondered if $A(X)$ is in fact generated by smooth rational surfaces. This has certainly been true for the few low-discriminant cases previously known, and the work here provides a lot more evidence for this possibility. 


\section{H. NUER}

Nevertheless, experimentation with $\mathcal{C}_{44}$ by generating surfaces on a cubic fourfold $X \in \mathcal{C}_{44}$ via residuation with the Fano Enriques surface $S$ has only given (smooth) surfaces of general type with fairly high degree. So it seems that the generic $X \in \mathcal{C}_{44}$ does not contain a smooth rational surface. Therefore, we are lead to ask the following.

Question 6.1. What does the condition that the generic $X \in \mathcal{C}_{d}$ contains a smooth rational surface not homologous to a complete intersection say, if anything, about the geometry of $X$ ? and about the geometry of $\mathcal{C}_{d}$ ?

\subsection{A note about the choices of $H$ in Table 1}

It seems fitting to end this section with a comment about how we came about the surfaces and embeddings given in Table 1. Completing the square in (3.4), we get

$$
\left(H^{2}-9\right)^{2}=81-d+3\left(3 H \cdot K_{S}+K_{S}^{2}-\chi_{S}\right)
$$

and from the assumption that $S$ is the blow-up of $\mathbb{P}^{2}$ at $p$ points and $H=a L-\left(E_{1}+\cdots+E_{i}\right)-$ $2\left(E_{i+1}+\cdots+E_{i+j}\right)-3\left(E_{i+j+1}+\cdots+E_{p}\right)$, we see that

$$
\begin{aligned}
H^{2} & =a^{2}-i-4 j-9(p-i-j), \\
H \cdot K_{S} & =-3 a+i+2 j+3 k .
\end{aligned}
$$

Combining (6.1) with the facts that $K_{S}^{2}=9-p$ and $\chi_{S}=3+p$, we see that

$$
\left(a^{2}-i-4 j-9(p-i-j)-9\right)^{2}=99-d+21 p-9(3 a+2 i+j) .
$$

This equation puts serious constraints on the non-negative integers $a, p, i, j$ and allows us to solve for $a$ in terms of the other three values. We note that automatically

$$
H^{2}\left(\mathcal{O}_{S}(H)\right)^{\vee}=H^{0}\left(\mathcal{O}_{S}\left(K_{S}-H\right)\right)=0,
$$

so $h^{0}\left(\mathcal{O}_{S}(H)\right)=\chi(H)+h^{1}\left(\mathcal{O}_{S}(H)\right)$. While not a necessary condition, it is reasonable to assume that the embedding $S \hookrightarrow \mathbb{P}^{5}$ is linearly normal and given by the complete linear system $|H|$, so that $h^{0}\left(\mathcal{O}_{S}(H)\right)=6$. We found the values in Table 1 by searching for non-negative integral solutions to (6.2) for each $d$ under the further assumption that $h^{1}\left(\mathcal{O}_{S}(H)\right)=0$ or, equivalently, that

$$
6=\chi\left(\mathcal{O}_{S}(H)\right)=\frac{1}{2}\left(H^{2}-H \cdot K_{S}\right)+1,
$$

which reduces the search to only two parameters. It is worth mentioning that this method does not seem to yield results for $d=42$ even upon relaxing these restrictions. Indeed, if we make mild assumptions on such a surface $S\left(\right.$ such as $\left.\left.h^{0}\left(\mathcal{O}_{S}(1)\right)\right) \geqslant 6, h^{0}\left(\mathcal{O}_{S}(2)\right) \geqslant 19, h^{0}\left(\mathcal{O}_{S}(3)\right) \leqslant 55\right)$, then the degree of the embedded surface is bounded above by 15 . Even allowing more complicated divisors, with coefficients on the $E_{i}$ larger than 3, produced either no non-negative integral solutions or solutions for which $\pi_{2}(\mathbf{F H})$ did not fill out $\mathcal{C}_{d}$. This certainly indicates that the method presented here has been exhausted for the most part.

\section{ACKNOWLEDGEMENTS}

I would like to thank my advisor, Lev Borisov, for his constant support and for encouraging me to push the limits of the methods developed here as far as possible. I would also like to thank Nick Addington for asking the question that essentially got this project started and for being a wonderful resource on cubic fourfolds. I thank Brendan Hassett for valuable discussions and in particular for placing these results in their proper context. I also benefited greatly from 


\section{UNIRATIONALITY OF MODULI SPACES}

discussions with Asher Auel, Anthony Várilly-Alvarado, and Michele Bolognesi. I would also like to thank an anonymous referee for reminding me about a result of Verra in [Ver84]. I am grateful to Mike Stillman and Dan Grayson for the program Macaulay2 [GS16], which was instrumental to the work here.

\section{REFERENCES}

CD85 F. Cossec and I. Dolgachev, Smooth rational curves on Enriques surfaces, Math. Ann. 272 (1985), no. 3, 369-384; https://doi.org/10.1007/BF01455565.

GHS13 V. Gritsenko, K. Hulek, and G. K. Sankaran, Moduli of K3 surfaces and irreducible symplectic manifolds, in Handbook of Moduli, Vol. I, Adv. Lect. Math. (ALM), vol. 24, eds G. Farkas and I. Morrison (Int. Press, Somerville, MA, 2013), 459-526.

GS16 D. Grayson and M. Stillman, Macaulay 2: A computer program designed to support computations in algebraic geometry and computer algebra, 2016, source and object code available from http: //www.math.uiuc.edu/Macaulay2/.

Has00 B. Hassett, Special cubic fourfolds, Compos. Math. 120 (2000), no. 1, 1-23; https://doi.org/ 10.1023/A:1001706324425.

Pet15 A. Peterson, Modular forms on the moduli space of polarised K3 surfaces, Ph.D. thesis, University of Amsterdam, 2015, available at http://hdl.handle.net/11245/2.162072.

Sch13 F. O. Schreyer, Computer aided unirationality proofs of moduli spaces, in Handbook of Moduli, Vol. III, Adv. Lect. Math. (ALM), vol. 26, eds G. Farkas and I. Morrison (Int. Press, Somerville, MA, 2013), 257-280.

TV15 S. Tanimoto and A. Várilly-Alvarado, Kodaira dimension of moduli of special cubic fourfolds, 2015, arXiv:1509.01562.

Uen75 K. Ueno, Classification theory of algebraic varieties and compact complex spaces, Lecture Notes in Math., vol. 439 (Springer-Verlag, Berlin - New York, 1975); https://doi.org/10.1007/ BFb0070570.

Ver84 A. Verra, A short proof of the unirationality of $\mathcal{A}_{5}$, Nederl. Akad. Wetensch. Indag. Math. 87 (1984), no. 3, 339-355; https://doi.org/10.1016/1385-7258(84)90033-7.

Voi07 C. Voisin, Some aspects of the Hodge conjecture, Jpn. J. Math. 2 (2007), no. 2, 261-296; https: //doi.org/10.1007/s11537-007-0639-x.

Voi14 On the universal $\mathrm{CH}_{0}$ group of cubic hypersurfaces, J. Eur. Math. Soc. (JEMS), to appear, arXiv:1407.7261.

Howard Nuer hjnuer@gmail.com

Department of Mathematics, Rutgers University, 110 Frelinghuysen Rd., Piscataway, NJ 08854, USA

Current address: Department of Mathematics, Statistics, and Computer Science, University of Illinois at Chicago, Chicago, IL 60607-7045, USA 